\title{
Suppression of SIPA-1 expression may reduce bladder cancer invasion and metastasis via the downregulation of E-cadherin and ZO-1
}

\author{
PING ZHANG ${ }^{1,2}$ and XINGHUAN WANG ${ }^{1}$ \\ ${ }^{1}$ Department of Urology, Zhongnan Hospital, Wuhan University, Wuhan, Hubei 430071; ${ }^{2}$ Department of Urology, \\ Yichang Central People's Hospital, The First Clinical Medical College, China \\ Three Gorges University, Yichang, Hubei 443003, P.R. China
}

Received July 14, 2014; Accepted June 5, 2015

DOI: 10.3892/etm.2015.2891

\begin{abstract}
The aim of the present study was to investigate the capacity of signal-induced proliferation-associated protein 1 (SIPA-1) to regulate bladder cancer cell invasion and metastasis. BIU-87 and T24 cells were transfected with the SIPA gene and SIPA short hairpin (sh)RNA, respectively. Western blot analysis was conducted to analyze the expression levels of SIPA-1, Ras-related protein 1 (Rap1), Rap1 guanosine triphosphate (Rap1GTP), E-cadherin and zona occludens-1 (ZO-1). Cell motility and invasion were evaluated in vitro using wound and Transwell assays. Transfected cells were inoculated into the pelvic region of $\mathrm{BALB} / \mathrm{c}$ nude mice, and the number of resulting tumors was recorded after 6 weeks. Western blot analysis revealed that expression levels of E-cadherin and ZO-1 were reduced in the cells with enhanced levels of SIPA-1. By contrast, the levels of E-cadherin and ZO-1 were elevated in the cells in which SIPA-1 was knocked down. In comparison with untransfected cells, the cells with reduced levels of SIPA-1 exhibited reduced wound closure and fewer cells crossed the chamber in the Transwell experiment, whereas the cells with enhanced levels of SIPA-1 exhibited increased migration and invasion In vivo, an increased tumor count was obtained in the mice with elevated levels of SIPA-1. Therefore, the results of the present study indicate that SIPA-1 is able to regulate bladder cancer cell metastasis and invasion by reducing the expression of E-cadherin and ZO-1.
\end{abstract}

\section{Introduction}

Bladder cancer is among the most common types of cancer worldwide, with $>330,000$ new cases reported each year and

Correspondence to: Dr Xinghuan Wang, Department of Urology, Zhongnan Hospital, Wuhan University, 169 Donghu Road, Wuhan, Hubei 430071, P.R. China

E-mail: zhongnan_wxh@sina.com

Key words: signal-induced proliferation-associated protein 1, invasion, metastasis, bladder cancer
$>130,000$ cases of mortality per year (1). At initial diagnosis, $30 \%$ of bladder cancer cases are diagnosed as muscle-invasive bladder cancer (MIBC) (2). A proportion of patients with MIBC require radical cystectomy in order to suppress cancer invasion and metastasis. However, the quality of life of an MIBC patient following radical cystectomy remains poor. Therefore, methods of suppressing MIBC progress to delay the necessity of surgery are urgently required.

Cancer metastasis comprises a series of complex processes and one of the initial steps of metastasis is the detachment and invasive migration of tumor cells from the primary tumor tissues. The extracellular matrix (ECM) serves a crucial function in this process (3). Ras-related protein (Rap) is a type of small GTP-binding protein that belongs to the Ras protein family. A number of studies have suggested that Rap signaling is closely associated with cell-ECM and cell-cell adhesion (4-6). Signal-induced proliferation associated protein-1 (SIPA-1, previously known as Spa-1) was originally isolated as a gene product of lymphoid cells activated by mitogenic stimulation (7). SIPA-1 is a regulator of Rap1, which is involved in Rap signaling (8). SIPA-1 is able to abrogate Rap1GTP, which is an active form of Rap1 that maintains cell adhesion, and then induce the detachment of cells from the matrix (9).

A number of previous studies have indicated that SIPA-1 is involved in certain processes associated with cancer activity, including mutation (10,11), apoptosis (12) and proliferation (13). Recent studies have indicated that SIPA-1 is associated with the motility of cancer cells (14-16). In addition, nuclear SIPA-1 activates the integrin $\beta 1$ promoter and promotes the invasion of breast cancer cells (15). SIPA-1 serves a variety of functions during disease progression and exerts contrasting effects on growth and motility of colorectal cancer cells (13). A previous study found that knockdown of SIPA-1 in colorectal cancer cells resulted in reduced cell growth in vitro; however, similar knockdown exhibited a contrasting effect on invasion and migration, which were increased in SIPA1-knockdown cells compared with the control cells (13). Furthermore, SIPA-1 knockdown regulated the invasion and metastasis of human prostate cancer (16). The Ras family includes Ras, Rap and Ral proteins, of which Ras and Ral have been demonstrated to have an association with bladder cancer $(17,18)$. 
The aim of the present study was to investigate the capacity of SIPA-1, a regulator of Rap, to regulate bladder cancer cell invasion and metastasis by modulating cell adhesion.

\section{Materials and methods}

Cell culture and reagents. T24 and BIU-87 cells were purchased from American Type Culture Collection (Manassas, VA, USA). Cells were grown in RPMI-1640 medium obtained from Life Technologies (Carlsbad, CA, USA) with 10\% fetal bovine serum at $37^{\circ} \mathrm{C}$ in an atmosphere of $95 \%$ air and $5 \%$ $\mathrm{CO}_{2}$. Monoclonal mouse anti-E-cadherin (\#14472) and rabbit anti-zona occludens-1 (ZO-1; \#14472) antibodies were purchased from Cell Signaling Technology, Inc. (Danvers, MA, USA). Monoclonal mouse anti-SIPA-1 (\#166219), anti-Rap1 (\#47695) and rabbit anti-GAPDH (\#365062) antibodies were purchased from Santa Cruz Biotechnology, Inc. (Santa Cruz, CA, USA).

Animals. A total of 48 male, 5-week-old BALB/c nude mice were purchased from the ABLS-3 laboratory of Wuhan University (Wuhan, China) and housed under standard conditions. All animal experiments were conducted in accordance with the UK Animals (Scientific Procedures) Act 1986 and associated guidelines, and the EEC Directive of 1986 (86/609/EEC). This study was approved by the Ethics Committee of Wuhan University School of Medicine.

Gene transfection. BIU-87 cells were transfected with a pcDNA3.1 vector containing the SIPA gene, or a control vector using opti-MEM (Life Technologies) and Lipofectamine 2000 (Life Technologies), and were selected using G418 reagent (Life Technologies) (16). The control vector consisted of the following sequence: 5'-GAT CCG TAC AGC GGT CCA ATC ATA GTA GTG CTC CTG GTT GCT ATG ATT GGA CCG CTG TAC TTT TTT AT-3'. SIPA-1 knockdown in T24 cells was achieved by transfecting the cells with a pSINsi-hU6 vector containing short hairpin (sh)RNA targeting SIPA1, or a control vector (16). The sequence of the shRNA for SIPA-1 was as follows: 5'-GAT CCG CTA CTT GCA ACA CCA TTC TTA GTG CTC CTG GTT GAG AAT GGT GTT GCA AGT AGC TTT TTT AT-3'. Transgene expression levels were determined using western blot analysis.

Western blot analysis for E-cadherin and ZO-1. The T24, control-T24, shRNA-SIPA-1-T24 (shT24), BIU-87, control-BIU-87 and SIPA-1-BIU-87 (S-BIU-87) cells were pooled and lysed using RIPA buffer. Samples of the lysates were resolved using SDS-PAGE and subjected to western blot analysis using standard procedures (19). The detection of intracellular Rap1GTP was conducted as described in a previous study (20).

Wound and Transwell assays. A wound assay was performed as previously described (21). A Transwell migration assay was conducted with the T24, control-T24 and shT24 cells using a QCM 24-well colorimetric cell migration assay kit (EMD Millipore, Billerica, MA, USA), following the manufacturer's instructions. BIU-87, control-BIU-87 and S-BIU-87 cells were also investigated using the same series of experiments.
The invasion assay was conducted in the same manner as the migration assay, but using a 24-well QCM ECMatrix Cell Invasion assay kit (EMD Millipore), based on the principle of the Boyden chamber. The results of the invasion assay were determined by observing six random fields at x200 magnification, and each experiment was repeated three times.

In vivo metastasis assay. The bladder cancer cells were diluted to $1 \times 10^{7} / 200 \mu 1$. Subsequently, 200- $\mu 1$ cell mixtures were inoculated into nude mice in the pelvic region. Mice were divided into six groups, with each receiving a different cell type: Group I, T24 cells; group II, control-T24 cells; group III, shT24 cells; group A, BIU-87 cells; group B, control-BIU-87 cells; and group C, S-BIU-87 cells $(n=8$ mice per group). The mice were sacrificed at 6 weeks after inoculation via intraperitoneally administered sodium pentobarbital $(60 \mathrm{mg} / \mathrm{kg})$. Resulting tumors were removed for assay.

Statistical analysis. SPSS software for Windows, version 17.0 (SPSS, Inc., Chicago, IL, USA) was used for statistical analysis. Data are presented as the mean \pm standard error of the mean. Statistical analysis was performed using unpaired t-test and one-way analysis of variance was used in comparisons of $>2$ groups. $\mathrm{P}<0.05$ was considered to indicate a statistically significant difference.

\section{Results}

Western blot analysis of E-cadherin and ZO-1 in T24 and BIU-87 cells. Western blot analysis indicated that the expression levels of E-cadherin and ZO-1 were increased in the shT2 4 cells, in which SIPA-1 expression was suppressed (Fig. 1A). By contrast, the levels of E-cadherin and ZO-1 were reduced in the S-BIU-87 cells, which were SIPA-1 enriched (Fig. 1B). Furthermore, the results indicate that the expression levels of Rap1GTP were negatively regulated by SIPA-1 (Fig. 1).

Wound and Transwell assays for T24 and BIU-87 cell migration and invasion. Compared with T24 cells, the shT24 cells with reduced expression of SIPA-1 showed decreased migration, leaving the majority of the wound area open (Fig. 2A). Conversely, S-BIU-87 cells with enhanced levels of SIPA-1 exhibited increased wound healing (Fig. 2B).

Compared with the T24 and control-T24 cells, markedly fewer cells crossed the Transwell chamber in the shT24 cell group. In the invasion assay, the shT24 cells that invaded through the Matrigel membrane were reduced in number (Fig. 3A). By contrast, the S-BIU-87 cells enriched in SIPA-1 crossed the chamber in greater numbers in the migration and invasion experiments (Fig. 3B).

Expression of SIPA-1 regulates bladder cancer cell metastasis in vivo. Bladder cancer cells were inoculated into the pelvic region of nude mice. After 6 weeks, the mice were sacrificed. Mice of groups I and II exhibited significantly increased numbers of tumors compared with the SIPA-1-silenced mice of group III $(25.5 \pm 5.35$ and $24.7 \pm 6.32$ vs. $10.3 \pm 3.66$, respectively; Fig. 4A). In the mice of groups $\mathrm{A}$ and $\mathrm{B}$, the numbers of tumors were significantly lower compared with those in 
A

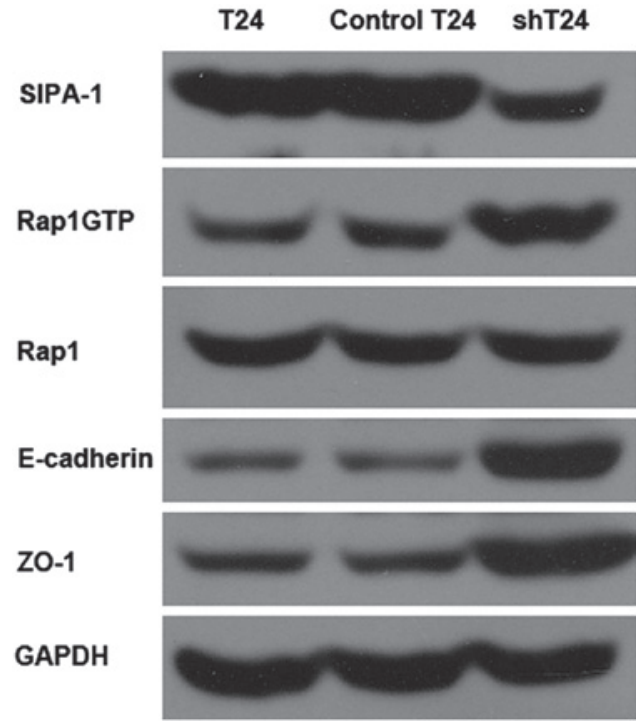

B

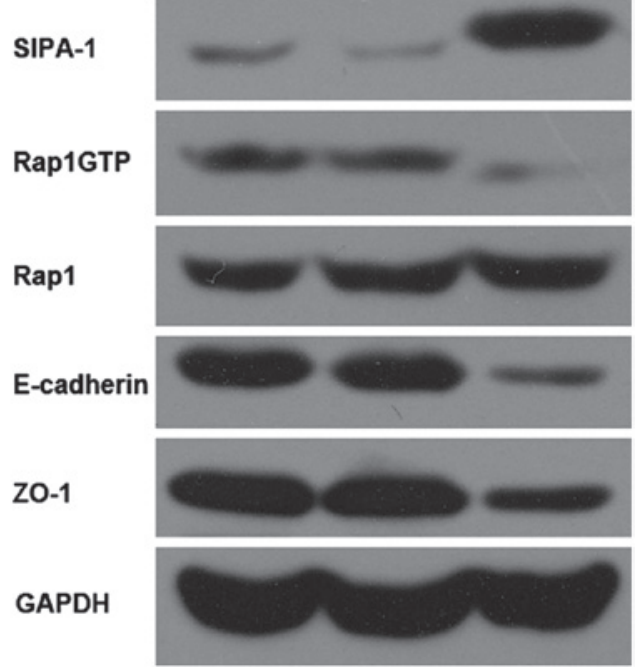

Figure 1. Western blot analysis of E-cadherin and ZO-1 in T24 and BIU-87 cells. Western blot analysis of the expression levels of SIPA-1, Rap1, Rap1GTP, E-cadherin and ZO-1 in (A) T24 and (B) BIU-87 cells and their transfected forms. Experiments were repeated six times. shT24, T24 cells transfected with short hairpin RNA targeting SIPA-1; S-BIU-87, BIU-87 cells transfected with the SIPA-1 gene. SIPA-1, signal-induced proliferation-associated protein 1; Rap1, Ras-related protein 1; ZO-1, zona occludens-1.
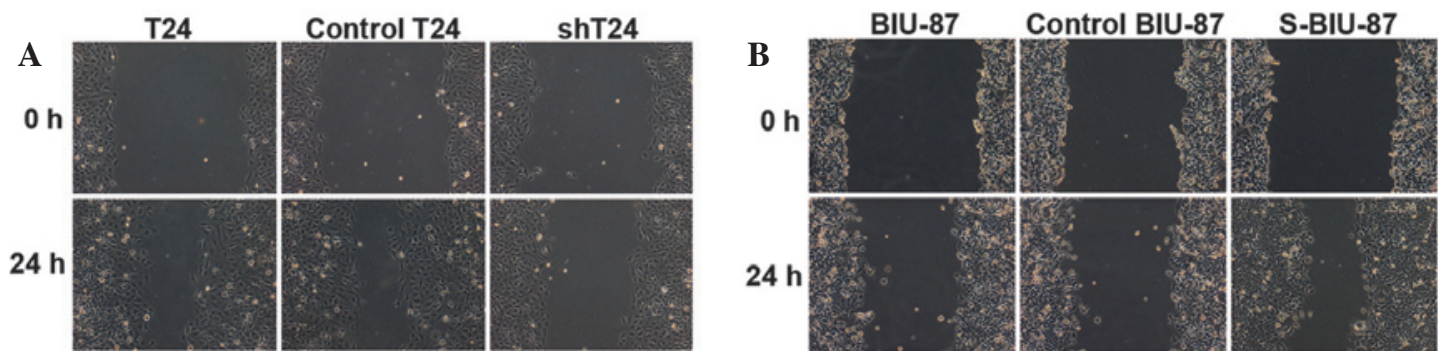

Figure 2. Wound and Transwell assays for T24 and BIU-87 cell migration and invasion. The metastasis of bladder cancer cells and transfected cells was measured using (A and B) wounding experiments. shT24, T24 cells transfected with short hairpin RNA targeting SIPA-1; S-BIU-87, BIU-87 cells transfected with the SIPA-1 gene. SIPA-1, signal-induced proliferation-associated protein 1.

the SIPA-1-overexpressing mice of group C $(2.13 \pm 1.4$ and $1.93 \pm 1.21$ vs. $7.85 \pm 3.5$, respectively; Fig. 4B).

\section{Discussion}

Metastasis is a type of cell activity that involves cell adhesion and motility. Therefore, the ability to control cancer cell adhesion may enable cancer cell metastasis to be reduced. Rap1 is a member of the Ras family of GTPases, and may serve a crucial function in the regulation of cell adhesion, depending on the cellular context (6). In contrast with Ras, which is active on the cell surface, Rapl activation is initiated within the cell and then migrates toward the cell surface (22). Rap1 is rapidly activated in adherent cells, including cancer cells, as a result of contact with ECM. Furthermore, basal Rap1 activation appears to be required for the maintenance of cell adhesion, as the conditional overexpression of SIPA-1 in adherent cells abrogates basal Rap1GTP and induces the detachment of cells from the ECM (9). The results of the present study indicate that Rap1GTP is associated with the expression levels of SIPA-1, and that metastatic cancer cells have elevated expression levels of SIPA-1 compared with primary cancer cells (Fig. 1).
The SIPA-1 family consists of numerous structurally-related but distinct proteins, including SIPA-1, E6-targeted protein 1 (E6TP1), spine-associated RapGAP (SPAR), and a number of SPA-1-like proteins (SPA-Ls), each presenting with a unique cellular distribution in various tissues (6). Previous studies have suggested that SIPA-1 is involved in certain types of cancer activity, including mutation $(23,24)$, apoptosis $(12,25)$, proliferation $(4,15)$, invasion $(15,16)$ and metastasis $(13,26,27)$. In the present study, it was observed that SIPA-1 additionally regulates bladder cancer cell metastasis and invasion (Figs. 2 and 3). Rap1GTP was upregulated, while E-cadherin and ZO-1 were upregulated as a result of the suppression of SIPA-1 (Fig. 1). E-cadherin is a member of the cadherin family, which is related to the transmembrane glycoproteins responsible for the $\mathrm{Ca}^{2+}$ dependent cell-cell adhesion mechanism that is crucial for the mutual association of vertebrate cells. A previous study demonstrated that impairment of the E-cadherin-mediated adhesion system is characteristic of cells with malignant transformation (28).

Rap is well known as a critical regulator of integrin-mediated cell adhesion; however, a previous study showed that Rap is crucially involved in the regulation of cadherin-mediated cell-cell adhesion in epithelial cells (29). 

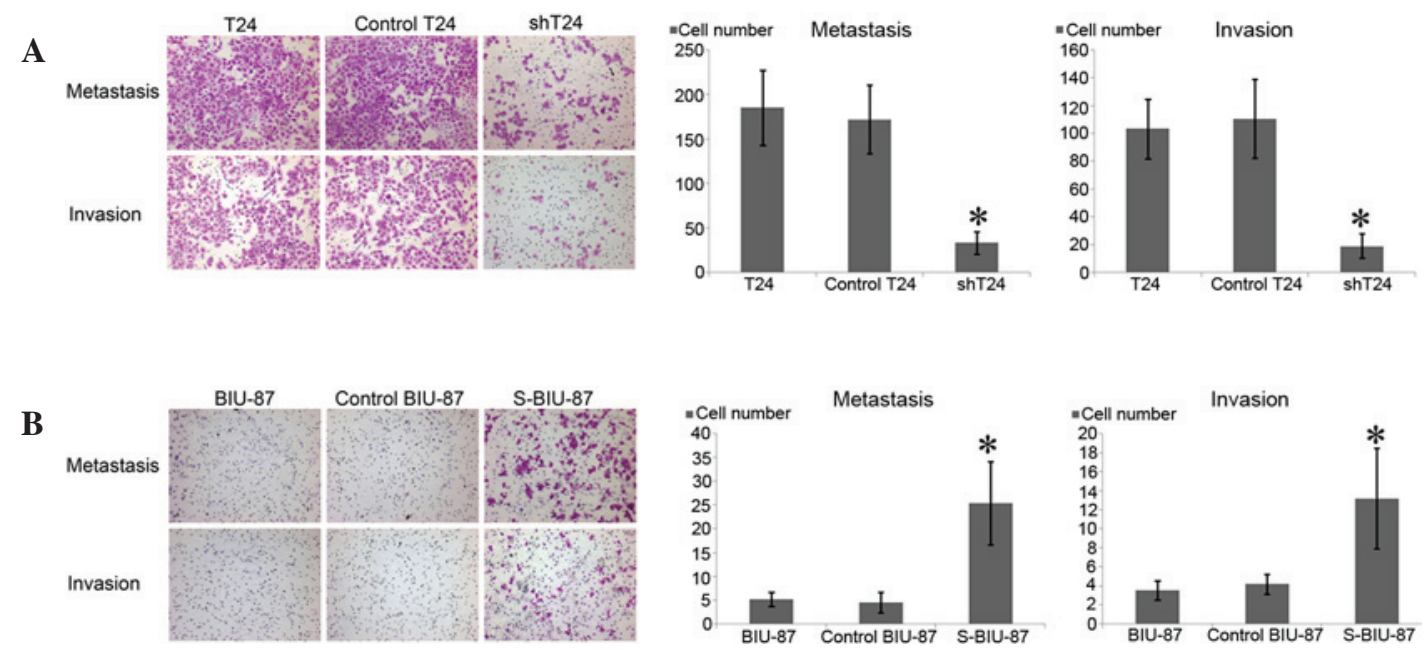

Figure 3. (A and B) The invasion of these cells was measured using Transwell assays with Matrigel. Results are the mean \pm standard deviation for six independent experiments; "P<0.05 vs. control and T24/BUI-87. shT24, T24 cells transfected with short hairpin RNA targeting SIPA-1; S-BIU-87, BIU-87 cells transfected with the SIPA-1 gene. SIPA-1, signal-induced proliferation-associated protein 1.

A

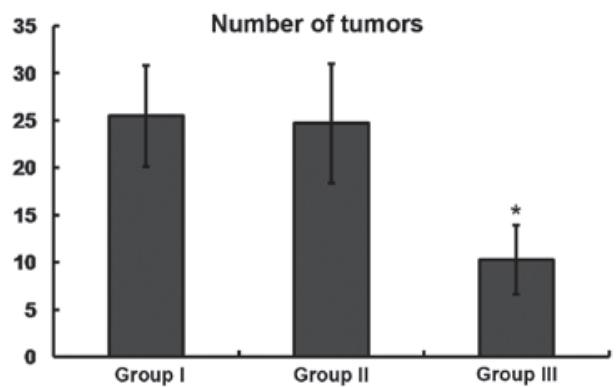

B

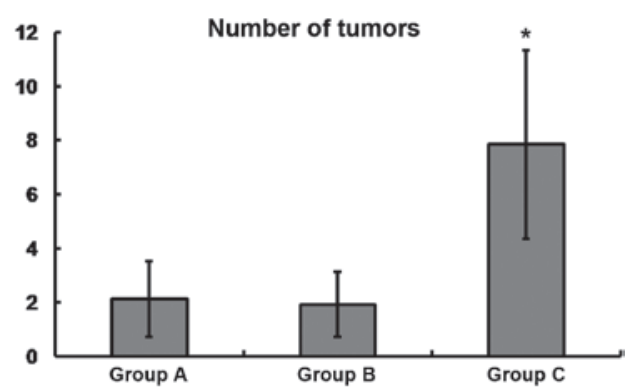

Figure 4. Expression levels of SIPA-1 appeared to regulate bladder cancer cells metastasis in an in vivo experiment. Mice were divided into 6 groups (n=8 mice per group). Group I, T24 cell mice; group II, control-T24 cell mice; group III, shT24 cell mice; group A, BIU-87 cell mice; group B, control-BIU-87 cell mice; and group C, SIPA-1-BIU-87 cell mice. Results are expressed as the mean \pm standard deviation. (A) * $\mathrm{P}<0.05$ vs. groups I and II and (B) * $\mathrm{P}<0.05$ vs. groups $\mathrm{A}$ and B. shT24, T24 cells transfected with short hairpin RNA targeting SIPA-1; S-BIU-87, BIU-87 cells transfected with the SIPA-1 gene. SIPA-1, signal-induced proliferation-associated protein 1 .

Furthermore, it has previously been demonstrated that the important role of Rap in homotypic E-cadherin interaction is independent of the effects of Rap on integrins (29). In the present study, T24 cells (with reduced SIPA-1 expression) and BIU-87 cells (with elevated SIPA-1 expression) were employed to investigate the association between SIPA-1 and the invasion and metastasis of bladder cancer cells, in addition to the expression of E-cadherin. Western blot analysis of the T24 and BIU-87 cells indicated that E-cadherin was negatively regulated by SIPA-1 (Fig. 1). The molecular mechanisms underlying the SIPA-1-mediated regulation of E-cadherin remain unclear, and it has been hypothesized that the protein afadin serves a pivotal function in the dynamic cyclical activation and inactivation of Rap1 via the coordinated regulation of SIPA-1 $(24,30)$.

$\mathrm{ZO}-1$ encodes a protein located on the cytoplasmic membrane surface of intercellular tight junctions. The encoded protein may be involved in signal transduction at cell-cell junctions. A previous study reported that the upregulation of ZO-1 correlates with the improved survival of gastrointestinal stromal tumor cells (31). In the present experiments, ZO-1 was negatively regulated by SIPA-1, in a similar manner to E-cadherin (Fig. 1). Previous studies have suggested that the
SIPA-1-induced downregulation of ZO-1 may involve afadin as a part of its underlying mechanism $(32,33)$.

The present study is an initial investigation into the role played by SIPA-1 in bladder cancer, and there were a number of limitations to this study. For example, the relation between SIPA-1 and MIBC survival curve will be imortant for investigat SIPA-1. The present study assessed the effects of SIPA-1 on a limited number of functions in bladder cancer. Future studies are required to evaluate the impact of SIPA-1 on cell proliferation and apoptosis, and the mechanistic association between afadin and SIPA-1 also requires elucidation. In the present in vivo experiment, metastasis of the cancer cells was reduced following the suppression SIPA-1 expression (Fig. 4). However, the present animal model may not be the most appropriate model for indicating clinical outcomes, and cancer cells inoculated in situ of the bladder may provide a more accurate model.

In conclusion, SIPA-1 has been demonstrated to regulate the metastasis and invasion of bladder cancer cells. This may be achieved by the negative regulation of the expression levels of E-cadherin and ZO-1. This insight may lead to the identification of novel therapy targets for the treatment of bladder cancer. 


\section{Acknowledgements}

This study was supported in part by a grant from the National Natural Science Foundation of China (no. 81172734).

\section{References}

1. Ploeg M, Aben KK and Kiemeney LA: The present and future burden of urinary bladder cancer in the world. World J Urol 27: 289-293, 2009.

2. Vaidya A, Soloway MS, Hawke C, Tiguert R and Civantos F: De novo muscle invasive bladder cancer: Is there a change in trend? J Urol 165: 47-50, 2001.

3. Friedl $\mathrm{P}$ and Wolf $\mathrm{K}$ : Tumour-cell invasion and migration: Diversity and escape mechanisms. Nat Rev Cancer 3: 362-374, 2003

4. Minato N: Rap G protein signal in normal and disordered lymphohematopoiesis. Exp Cell Res 319: 2323-2328, 2013.

5. Minato N and Hattori M: Spa-1 (Sipa1) and Rap signaling in leukemia and cancer metastasis. Cancer Sci 100: 17-23, 2009.

6. Kometani K, Ishida D, Hattori $\mathrm{M}$ and Minato N: Rap1 and SPA-1 in hematologic malignancy. Trends Mol Med 10 401-408, 2004.

7. Hattori M, Tsukamoto N, Nur-e-Kamal MS, Rubinfeld B, Iwai K Kubota $\mathrm{H}$, Maruta $\mathrm{H}$ and Minato N: Molecular cloning of a novel mitogen-inducible nuclear protein with a Ran GTPase-activating domain that affects cell cycle progression. Mol Cell Biol 15: $552-560,1995$

8. Kurachi H, Wada Y, Tsukamoto N, Maeda M, Kubota $\mathrm{H}$, Hattori M, Iwai $\mathrm{K}$ and Minato N: Human SPA-1 gene product selectively expressed in lymphoid tissues is a specific GTPase-activating protein for Rap1 and Rap2. Segregate expression profiles from a rap1GAP gene product. J Biol Chem 272: 28081-28088, 1997.

9. Tsukamoto N, Hattori M, Yang H, Bos JL and Minato N: Rap1 GTPase-activating protein SPA-1 negatively regulates cell adhesion. J Biol Chem 274: 18463-18469, 1999.

10. Kim T, Noh M, Lee H, Joo SW, Lee SY and Lee K: Fluorescence-based detection of point mutation in DNA sequences by CdS quantum dot aggregation. J Phys Chem B 113: 14487-14490, 2009.

11. Yoshida N, Yagasaki H, Takahashi Y, Kudo K, Manabe A and Kojima S: Mutation analysis of SIPA1 in patients with juvenile myelomonocytic leukemia. Br J Haematol 142: 850-851, 2008.

12. Katagiri K, Hattori M, Minato N and Kinashi T: Rap1 functions as a key regulator of T-cell and antigen-presenting cell interactions and modulates T-cell responses. Mol Cell Biol 22: 1001-1015, 2002.

13. Ji K, Ye L, Toms AM, Hargest R, Martin TA, Ruge F, Ji J and Jiang WG: Expression of signal-induced proliferation-associated gene 1 (SIPA1), a RapGTPase-activating protein, is increased in colorectal cancer and has diverse effects on functions of colorectal cancer cells. Cancer Genomics Proteomics 9: 321-327, 2012.

14. Brooks R, Kizer N, Nguyen L, Jaishuen A, Wanat K, Nugent E, Grigsby P, Allsworth JE and Rader JS: Polymorphisms in MMP9 and SIPA 1 are associated with increased risk of nodal metastases in early-stage cervical cancer. Gynecologic Oncol 116: 539-543, 2010.

15. Zhang Y, Zhang Y, Gong Y, Hu D1, Zhu P, Wang N, Zhang Q, Wang M, Aldeewan 1, Xia H, Qu X, et al: Nuclear SIPA1 activates integrin betal promoter and promotes invasion of breast cancer cells. Oncogene 34: 1451-1462, 2014.

16. Shimizu Y, Hamazaki Y, Hattori M, Doi K, Terada N, Kobayashi T, Toda Y, Yamasaki T, Inoue T, Kajita Y, et al: SPA-1 controls the invasion and metastasis of human prostate cancer. Cancer Sci 102: 828-836, 2011.
17. Saito R, Shirakawa R, Nishiyama H, Kobayashi T, Kawato M, Kanno T, Nishizawa K, Matsui Y, Ohbayashi T, Horiguchi M, et al: Downregulation of Ral GTPase-activating protein promotes tumor invasion and metastasis of bladder cancer. Oncogene 32: 894-902, 2013

18. Smith SC, Baras AS, Owens CR, Dancik G and Theodorescu D: Transcriptional signatures of Ral GTPase are associated with ag gressive clinicopathologic characteristics in human cancer. Cancer Res 72: 3480-3491, 2012.

19. Roomi MW, Kalinovsky T, Cha J, Roomi NW, Niedzwiecki A and Rath M: Effects of a nutrient mixture on immunohistochemical localization of cancer markers in human cervical cancer HeLa cell tumor xenografts in female nude mice. Exp Ther Med 9 294-302, 2015.

20. Reedquist KA and Bos JL: Costimulation through CD28 suppresses $T$ cell receptor-dependent activation of the Ras-like small GTPase Rapl in human T lymphocytes. J Biol Chem 273: 4944-4949, 1998.

21. Wang Y, Wang X, Yang Z, Zhu G, Chen D and Meng Z: Menthol inhibits the proliferation and motility of prostate cancer DU145 cells. Pathol Oncol Res 18: 903-910, 2012.

22. Mochizuki N, Yamashita S, Kurokawa K, Ohba Y, Nagai T, Miyawaki A and Matsuda M: Spatio-temporal images of growth-factor-induced activation of Ras and Rap1. Nature 411: 1065-1068, 2001

23. Tanaka H, Tamura A, Sekai M, Hamazaki Y and Minato N: Increased c-Myc activity and DNA damage in hematopoietic progenitors precede myeloproliferative disease in Spa-1-deficiency. Cancer Sci 102: 784-791, 2011.

24. Miyata M, Rikitake Y, Takahashi M, Nagamatsu Y, Yamauchi Y, Ogita H, Hirata K and Takai Y: Regulation by afadin of cyclical activation and inactivation of Rap1, Racl, and RhoA small $\mathrm{G}$ proteins at leading edges of moving NIH3T3 cells. J Biol Chem 284: 24595-24609, 2009.

25. Crawford NP, Qian X, Ziogas A, Papageorge AG, Boersma BJ, Walker RC, Lukes L, Rowe WL, Zhang J, Ambs S, et al: Rrplb, a new candidate susceptibility gene for breast cancer progression and metastasis. PLoS Genet 3: e214, 2007.

26. Roberts MR, Hong CC, Edge SB, Yao S, Bshara W, Higgins MJ, Freudenheim JL and Ambrosone CB: Case-only analyses of the associations between polymorphisms in the metastasis-modifying genes BRMS1 and SIPA1 and breast tumor characteristics, lymph node metastasis, and survival. Breast Cancer Res Treat 139: 873-885, 2013.

27. Alsarraj J, Faraji F, Geiger TR, Mattaini KR, Williams M, Wu J, Ha NH, Merlino T, Walker RC, Bosley AD, et al: BRD4 short isoform interacts with RRP1B, SIPA1 and components of the LINC complex at the inner face of the nuclear membrane. PLoS One 8: e80746, 2013.

28. Shiozaki H, Oka H, Inoue M, Tamura S and Monden M: E-cadherin mediated adhesion system in cancer cells. Cancer 77 (Suppl): 1605-1613, 1996.

29. Price LS, Hajdo-Milasinovic A, Zhao J, Zwartkruis FJ, Collard JG and Bos JL: Rap1 regulates E-cadherin-mediated cell-cell adhesion. J Biol Chem 279: 35127-35132, 2004.

30. Mandai K, Nakanishi H, Satoh A, Obaishi H, Wada M, Nishioka H, Itoh M, Mizoguchi A, Aoki T, Fujimoto T, et al: Afadin: A novel actin filament-binding protein with one PDZ domain localized at cadherin-based cell-to-cell adherens junction. J Cell Biol 139: 517-528, 1997.

31. Zhu H, Lu J, Wang X, Zhang H, Tang X, Zhu J and Mao Y: Upregulated ZO-1 correlates with favorable survival of gastrointestinal stromal tumor. Med Oncol 30: 631, 2013.

32. Birukova AA, Fu P, Wu T, Dubrovskyi O, Sarich N, Poroyko V and Birukov KG: Afadin controls p120-catenin-ZO-1 interactions leading to endothelial barrier enhancement by oxidized phospholipids. J Cell Physiol 227: 1883-1890, 2012.

33. Severson EA, Lee WY, Capaldo CT, Nusrat A and Parkos CA Junctional adhesion molecule A interacts with Afadin and PDZ-GEF2 to activate Rap1A, regulate beta1 integrin levels, and enhance cell migration. Mol Biol Cell 20: 1916-1925, 2009. 